\title{
100 Jahre Euphorbia handiensis, Teil II: Bedrohung und Schutz
}

\author{
Hans Grasmück \& Stephan Scholz
}

\begin{abstract}
Summary
The endangerment of the spurge Euphorbia handiensis from Fuerteventura, mainly caused by extensive goat keeping, is pointed out. Precautionary measures are suggested and problems discussed.
\end{abstract}

\section{Zusammenfassung}

Es wird auf die Gefährdung des Wolfsmilchgewächses Euphorbia handiensis aus Fuerteventura hingewiesen, die schwerpunktmäßig durch die dortige Ziegenhaltung bedingt ist. Es werden Schutzmaßnahmen vorgeschlagen und Probleme bei ihrer Umsetzung diskutiert.

\section{Bedrohungen der Bestände von Euphorbia handiensis}

In der vorigen Ausgabe wurde über das Auffinden und die Vergesellschaftung von Euphorbia handiensis, Lokalendemit von Fuerteventura, berichtet (Grasmück \& Scholz 2013). Nach ihrer Entdeckung gab es über einen Zeitraum von gut 60 Jahren kaum Nachrichten über den Zustand und die Entwicklung der Populationen. Burchard selbst $(1912,1929)$ sah die Art nicht als bedroht an. Er hinterließ aber das einzige uns bekannte Zeugnis, das für die Einschätzung der längerfristigen Bestandsentwicklung herangezogen werden kann: das auch von Lavant \& Suntjens (2006) erwähnte Foto einer sehr großen, flächendeckenden Population der Art am südlichsten Ende des Bergzuges $\mathrm{Cu}$ chillo del Palo, in der Nähe des Talausgangs des Valle de los Mosquitos. Hier stehen heute nur ganz vereinzelt Exemplare; über lange Strecken gibt es gar keine mehr. Die Ursachen dafür sind nicht geklärt. Es kommen verschiedene Möglichkeiten bzw. Kombinationen davon infrage, die weiter unten diskutiert werden.

Die lange Zeit sehr spärlicher Informationen über $E$. handiensis verstehen sich vor allem aus der Abgelegenheit ihres Wuchsgebietes. Jandia war nur mühselig zu erreichen, seit der Eroberung der Insel im frühen 15. Jh. in Privathand und zeitweilig (1937 - 1964) sogar fast vollständig von der Außenwelt abgeschottet. Dennoch konnten Forscher wie ErIC R. Sventenius in den Vierziger- und Fünfziger-Jahren des vorigen Jahrhunderts das Gebiet mit Erlaubnis des Pächters bzw. Eigentümers besuchen. Aber erst in neuerer Zeit, nach der Teilung des über 550 Jahre zusammenhängenden Großgrundbesitzes und der Öffnung für die touristische Entwicklung, wurde dieser abgelegene Inselteil wieder regelmäßig von Botanikern aufgesucht.

Die ersten neueren Angaben über E. handiensis (Bramwell \& Bramwell 1974, Kunkel 1977) weisen bereits auf die Gefährdung der Art hin, wobei meist die negativen Folgen der Urbanisation und das Sammeln der Pflanzen für den Gartenbau als wichtigste Bedrohungen angesehen werden, daneben die Zerstörung der Samen durch Insekten. Diese Aussagen änderten sich in der Folgezeit wenig (Gómez-CAmpo 1996, Beltrán Tejera et al. 1999). Eine Ausnahme machen Marrero \& Carqué (2003), die von ,einer klaren Erholung der Art in den letzten Jahren“" sprechen. Diese vermeintliche Erholung der Bestände ist zumindest teilweise eine Konsequenz verbesserter Untersuchungsmethodik sowie der Unterschätzung der Bestandszahlen in vorangegangenen Jahren. Die genannten Autoren geben einen Gesamtbestand von über 200000 Individuen an, viermal höher, als ihn Magnani (1988) fünfzehn Jahre vorher geschätzt hatte, der aber nur zwei der drei Lokalitäten von $E$. handiensis kannte.

Magnani (1992) ist unseres Wissens der Erste, der auf das Phänomen der abgebrochenen Zweige und zerstörten Exemplare der JandiaWolfsmilch hinweist. Lawant \& Suntjens (2006) gehen in ihrer ausführlichen Arbeit näher darauf ein und führen es auf die direkte Aktion der Ziegen zurück, die sie besonders im Sommer regelmäßig beim Zertrampeln von Ex- 


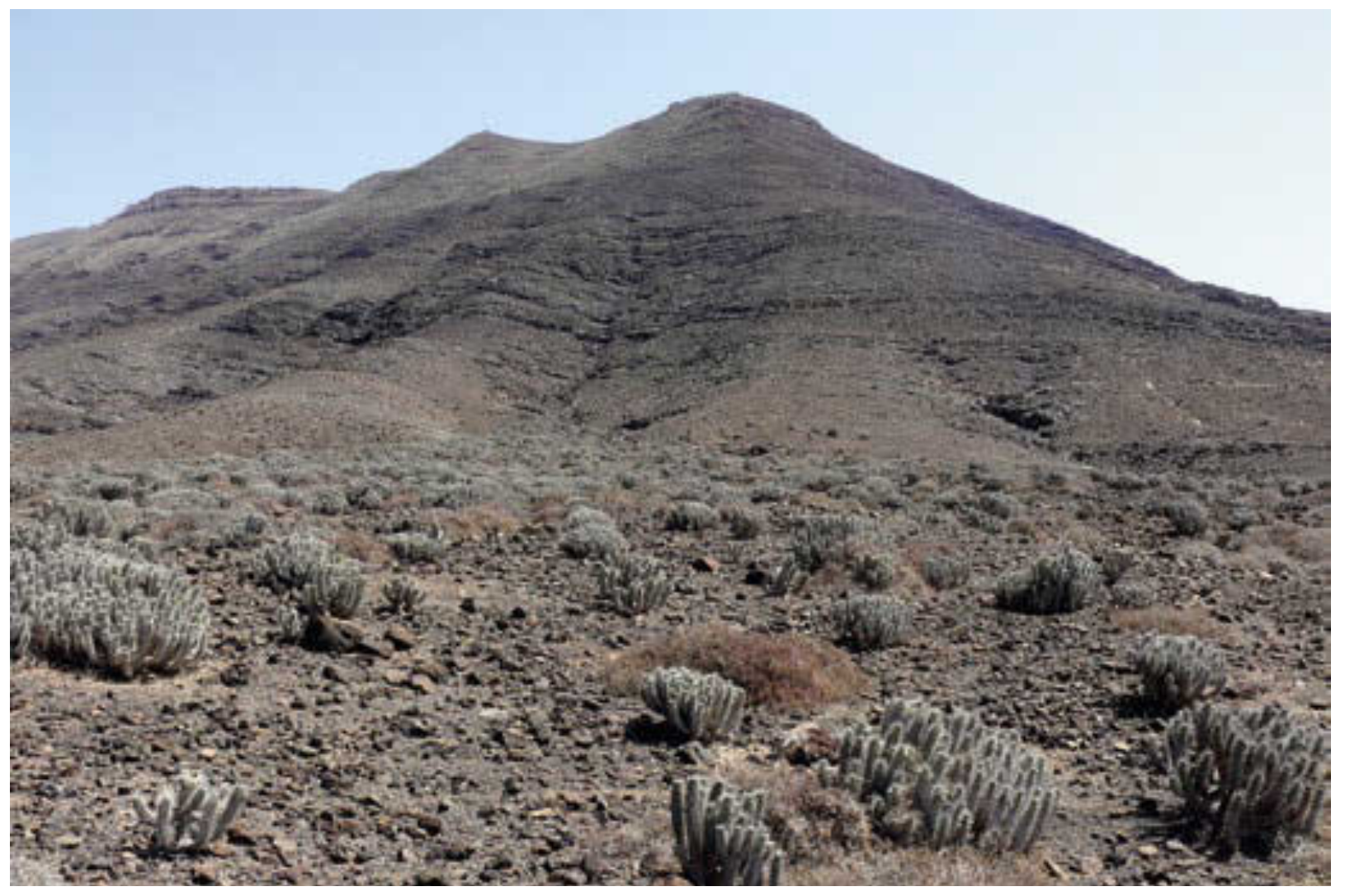

emplaren beobachteten, um an die im Inneren der Euphorbia-Büsche wachsenden Gräser und Kräuter heranzukommen. Der Zweitautor, der seit 27 Jahren ununterbrochen in Jandia lebt und arbeitet und viele Stunden im Gelände verbracht hat, konnte dies ebenso wie den auch geschilderten direkten Verzehr junger Euphorbia-Pflanzen durch Ziegen bisher nicht beobachten, möchte aber nicht bestreiten, dass beides vorkommen kann.

Das Sterben von Exemplaren aller Altersstufen ist an mehreren Orten festzustellen. Am auffälligsten ist es, wie Lawant \& Suntjens (2006) darlegen, im unteren Teil des Gran Valle und dort besonders im näheren Umkreis einer Ansammlung von Ziegengehegen und Hütten, die mindestens seit 1988 zeitweilig bewohnt sind. Im unteren Teil des Valle de Jorós ist das gehäufte Absterben von Exemplaren ebenso zu sehen. Dort befinden sich keine Krale, Ziegen

Abb 1: Der Gebirgskamm Cuchillo del Palo mit seiner Euphorbia-handiensis-Vegetation am Talausgang des Valle de los Mosquitos. durchstreifen das Gebiet aber wie überall in Jandia. Im Valle de los Mosquitos dagegen kommen nur vereinzelt abgestorbene Exemplare vor.

Wir führen den Zusammenbruch der Sträucher eher auf die Veränderung des Lebensraumes durch Zertrampeln der oberen Bodenschicht durch Ziegenhufe und mögliche Stickstoffbelastung des Bodens zurück, die sich durch die hohe Ziegendichte im Umkreis der Krale ergibt. Der viel bessere Zustand der Populationen von $E$. handiensis im Valle de los Mosquitos, das ebenfalls regelmäßig von Ziegen aufgesucht wird, könnte teilweise darauf zurückzuführen sein, dass der Boden hier im Gegensatz zum unteren Teil vom Gran Valle stärker von Steinen und Felsbrocken bedeckt ist. Dies schützt ihn vor Vertritt, hat eine bessere Wasserhaltung zur Folge und schafft in Nischen und Felsritzen außerdem geschützte Plätze zum Keimen der Jungpflanzen. Überhaupt sind die Bestände der Wolfsmilch in steilem, felsigen Gelände oft gesünder als auf den leicht zugänglichen, mit lehmigem Boden bedeckten Hangfüßen. Die Boden- und allgemeine Vegetationszerstörung durch Ziegen in den unteren 


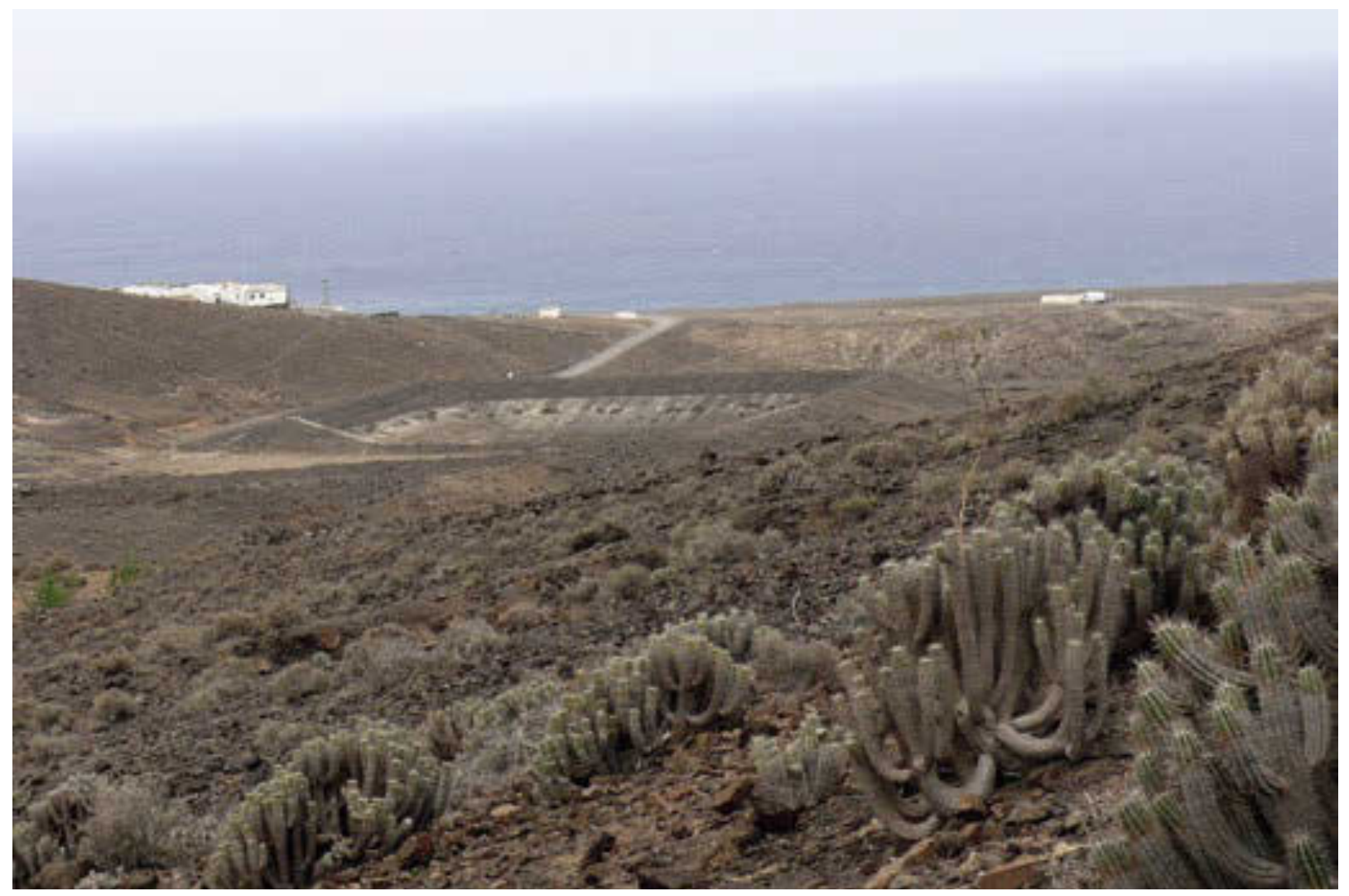

Bereichen des Gran Valle und Valle de Jorós hat unserer Meinung nach eine Schwächung der Wolfsmilch-Pflanzen zur Folge, die dann leichter von Pilzen, bakteriellen Erkrankungen oder Insekten befallen werden. Ohne die Möglichkeit der direkten Zerstörung der Pflanzen durch Ziegen auszuschließen, sind wir der Meinung, dass die äußeren Symptome vieler sterbender Wolfsmilch-Exemplare mehr auf Krankheitsbefall hindeuten, weil oft keine mechanischen Verletzungen festzustellen sind und die Pflanzen oder Teile von ihnen einfach verrotten und wegbrechen. Möglicherweise tragen leichte Verletzungen der oberflächlich verlaufenden Wurzeln oder Äste durch Ziegenhufe dazu bei, dass Krankheitserreger in die Pflanzen eindringen können. Vorläufige Untersuchungen in einem Labor auf Teneriffa, beantragt von der Abteilung Naturschutz der Inselverwaltung von Fuerteventura, ergaben die Anwesenheit von Phoma sp. und Coniothyrium sp. in abgestorbenen bzw. kranken Ästen der Jandia-Wolfsmilch. Es ist aber unklar, ob diese Pilze die primären Verursacher sind. Vereinzelt werden in sterbenden Exemplaren Bereiche mit nasser Fäulnis beobachtet, in denen Larven von Schwebfliegen (Syrphidae) gefunden wurden.

Die allgemeine Zerstörung der Vegetation vor allem an den ersten beiden Standorten von E. handiensis, also die "Bereinigung“ des Terrains von Gräsern, Kräutern und anderen Pflanzen durch Ziegen, hat noch eine andere bedeutungsvolle Konsequenz: es gibt im Winter und Frühjahr einen beträchtlichen Mangel an blühenden Pflanzen. Dies wiederum führt zum allmählichen Zusammenbruch der vielfältigen Insektenpopulation, die zur Ernährung Nektar benötigt. Wir haben an den Cyathien von $E$. handiensis vor allem verschiedene Fliegen- und

Abb. 2 (oben): Ein Bestand von Euphorbia handiensis am Ausgang des Valle de Jorós mit den Casas de Jorós und einem Wasserspeicher.

Abb. 3 (Seite 95 oben): Der Viehzuchtbetrieb La Solana im Gran Valle, im Vordergrund E. handiensis.

Abb. 4 (Seite 95 Mitte): Abgestorbenes Exemplar von $E$. handiensis.

Abb. 5 (Seite 95 unten): Durch mechanische Einwirkung zerstörte E. handiensis im Gran Valle. 
Ameisen-Arten beobachtet. Fehlen die Insekten, dann entfällt die Bestäubung der Blüten, und dies verhindert auch die Bildung fertiler Samen. Direkte Schäden durch Nagetiere kommen ebenfalls vor, sie sind aber relativ selten und treten nur im Sommer auf. Die Symptome sind unregelmäßig zerschlissene und zerfetzte Äste im nahen Bodenbereich. Das innere Gewebe wird gefressen. Die Pflanzen werden über mehrere Tage (oder Nächte) regelmäßig besucht und schließlich oft ganz zerstört. Wir fanden Verletzungen dieser Art zuerst auf einem privaten Gelände des Zweitautors, wo dieser versucht hatte, E. handiensis über aus Gärtnereien bezogene junge Pflanzen anzusiedeln. Da das Grundstück gegen Ziegen eingezäunt ist, kommen als Verursacher nur Wildkaninchen und nordafrikanische Streifenhörnchen (Atlantoxerus getulus) infrage. Letztere wurden in den 1960er-Jahren aus der Westsahara eingeführt, eroberten schnell die ganze Insel und wurden lokal zur Plage in der Landwirtschaft (Abfressen von Rinde an Feigenbäumen und anderen Pflanzen). Wir haben aber als Verursacher der Schäden an E. handiensis - die geschilderten Verletzungen wurden gelegentlich auch in den natürlichen Beständen der Wolfsmilch gefunden - eher Kaninchen in Verdacht. Sie sind im Gegensatz zu den oft mehr umherschweifenden Streifenhörnchen standorttreu und finden in den langen, trockenen Sommern wohl etwas Nahrung, aber kaum Feuchtigkeit. Das wasserspeichernde innere Gewebe der Wolfsmilch, weitgehend frei von Milchsaft, ist dabei ein willkommener Spender. Die Kaninchen vertilgen auch Kleinien (Kleinia neriifolia), trotz ihres Gehaltes an pyrrolizidinen Alkaloiden (Hansen 1997, Dominguez et al. 2008).

Kakteen und sogar Aloe vera bleiben auch nicht unbehelligt. Euphorbia canariensis wird manchmal "probeweise“ angebissen, anscheinend ist aber ihr Milchsaft zu stark ätzend, sodass der Fressversuch wieder aufgegeben wird. Schon Kunkel (1977) erwähnt, dass die Samen von $E$. handiensis durch Insekten zerstört werden. Bei Untersuchungen im Sommer 2011 fanden wir im Valle de los Mosquitos an den meisten Exemplaren zahlreiche befallene Sa-
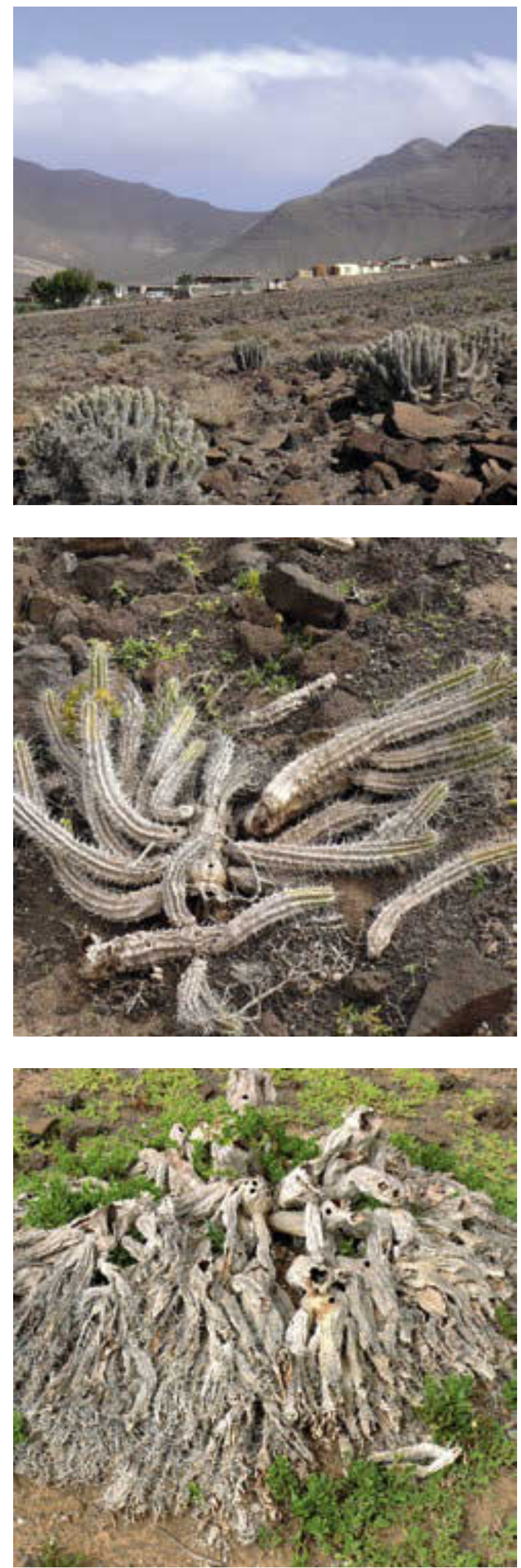

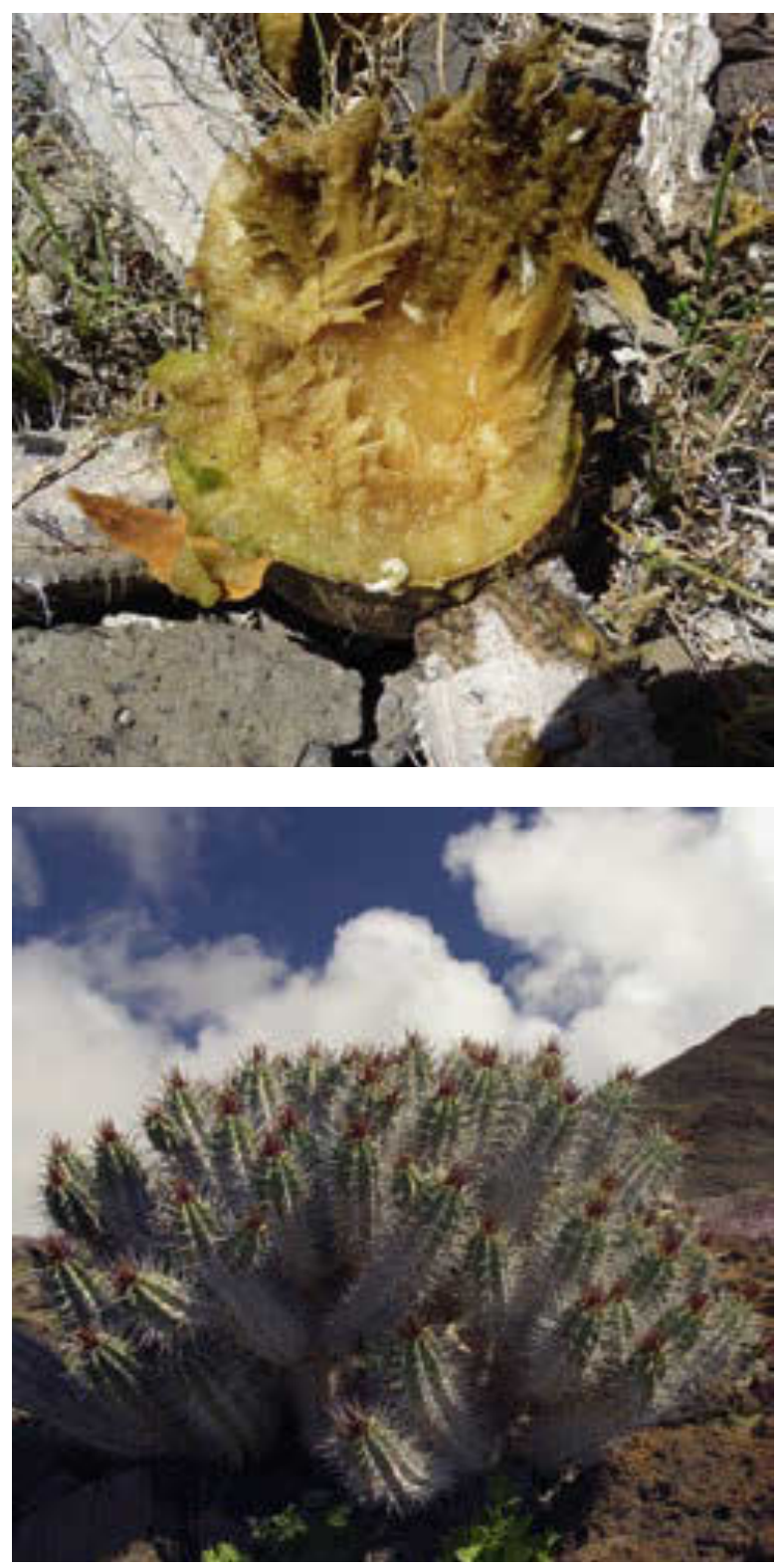

menkapseln. Aus winzigen orangefarbenen Raupen im Inneren der Kapseln gingen im Einmachglas ca. $4 \mathrm{~mm}$ lange Kleinschmetterlinge hervor, von denen bisher nur die Familie (Gelechidae) bestimmt werden konnte. Sich von Samen ernährende Larven von Gelechiden finden sich auf Fuerteventura auch auf E. balsamifera (bisher unbestimmt) und an E. canariensis. Hier handelt es sich um Streyella canariensis Walsingham (det. Wolfram Mey, Museum für Naturkunde, Berlin). Sicherlich gehören all diese Kleinschmetterlinge zu der natürlichen Insektenfauna der Euphorbien und können von deren Populationen verkraftet werden, solange diese nicht durch andere Faktoren zusätzlich geschwächt sind, wie es jetzt leider in großen Teilen des Verbreitungsgebietes von $E$. handiensis der Fall ist. Auch die Käferfauna an E. handiensis ist vielfältig. Es wurde z. B. Aphanartherum mairei (Curculionidae) in abgestorbenen Pflanzenteilen gefunden; die Art kommt auch in Marokko an E. echinus vor. Anscheinend sind aber noch keine spezifischen Samenschädlinge für $E$. handiensis bekannt.

Das Ausgraben von Exemplaren der JandiaWolfsmilch für den Gartenbau spielt heute, wie auch LaWAnt \& Suntjens (2006) feststellen, kaum eine Rolle. Gelegentliches Mitnehmen von jungen Pflanzen durch Touristen gefährdet die Bestände nicht, und planmäßiges Ausreißen in größerem Maßstab ist bei dieser offiziell geschützten Art riskant. Zehn „Agentes medioambientales“, von der Inselverwaltung angestellte Aufseher mit den Vollmachten eines Polizeibeamten, übernehmen z. Z. die Naturschutz-Aufsicht auf Fuerteventura, ausgestattet mit Geländewagen und unterstützt durch lokale Aufseher der Gemeinden. Eine größere Gefahr für die Bestände im unteren Teil des Gran Valle geht, wie Lawant \& Suntjens (2006) ebenfalls darlegen, von der möglichen Urbanisation des Gebietes aus. Ein schmaler sich von Morro Jable aus ca. $7 \mathrm{~km}$ nach Westen erstreckender Küstenstreifen ist von dem 14318 ha umfassenden Naturpark Jandia ausgeschlossen, um weitere touristische Entwicklung zu ermöglichen und auch Unterkünfte für die lokale Bevölkerung zu schaffen. Die der Küste am nächsten wachsenden Exemplare der Wolfsmilch im Gran Valle dürften ungefähr auf der Grenze zwischen geschütztem Gebiet und Bauland liegen. Die ungünstige wirtschaftliche Lage hat bisher verhindert, dass aus bereits bestehenden Bauplänen Realität wurde. Selbst wenn die Pflanzen nicht direkt durch die Baumaßnahmen betroffen würden, so hätte die unmittelba-

Abb. 6 (oben): Zerstörter, noch saftiger Trieb von $E$. handiensis.

Abb. 7 (unten): Attraktive Jungpflanzen.

Abb. 8 (Seite 97): Braune und eingetrocknete Triebe einer E. handiensis. 


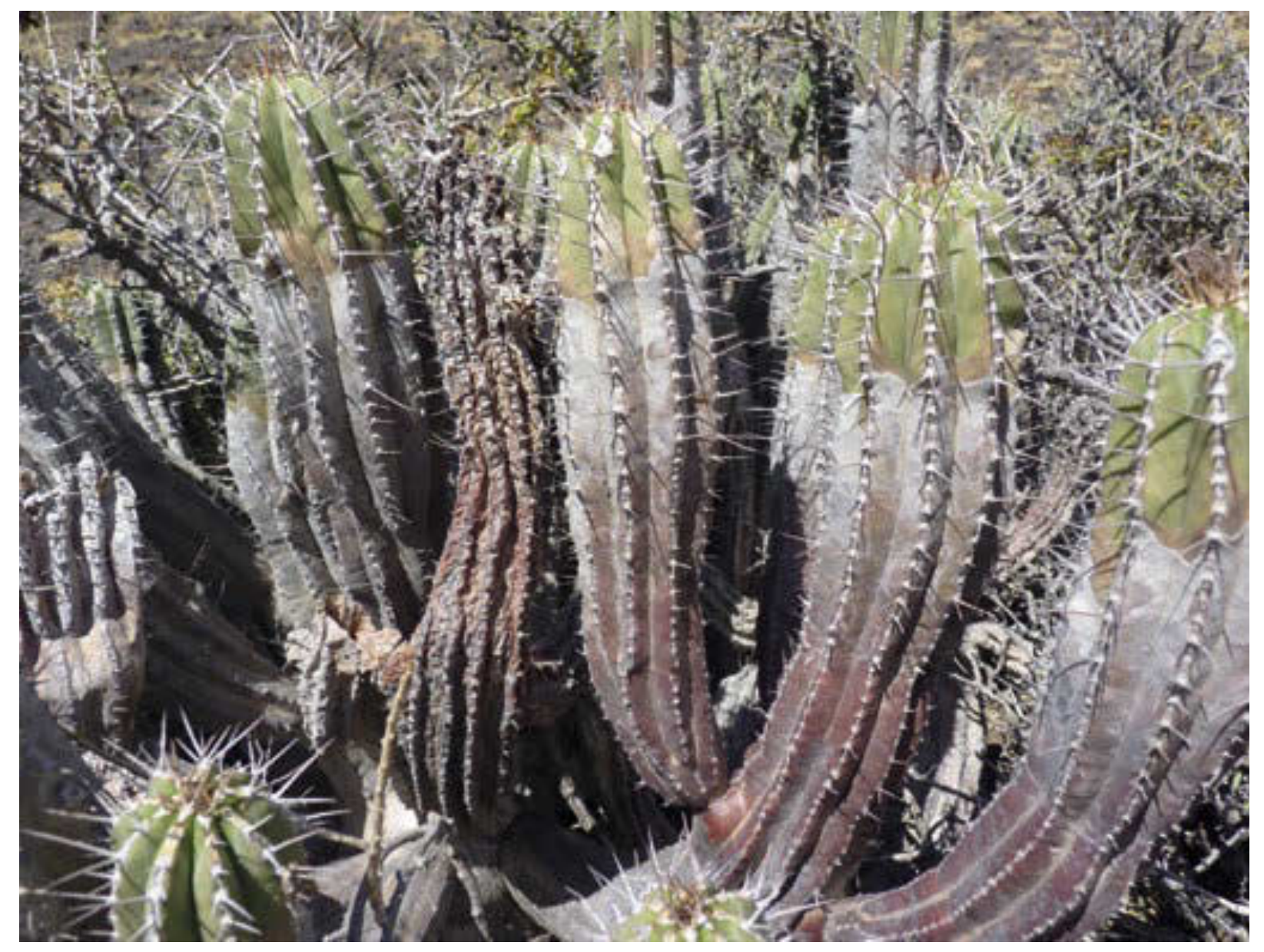

re Nähe von Urbanisationen mit Sicherheit zusätzliche negative Folgen für die Population.

Beim Untersuchen der verschiedenen möglichen Ursachen für den Rückgang der Bestände der Jandia-Wolfsmilch ist ein Faktor nicht beachtet worden: das Abschlagen und Sammeln von Sträuchern, um sie als Brennmaterial in Kalköfen zu verwenden. Diese großen, runden, aus Naturstein gebauten Öfen sind seit dem 16. Jh. auf Fuerteventura bekannt. In ihnen wurde bis vor 50-60 Jahren Kalk zum Bauen und zum Verputzen von Wänden gebrannt, der in großem Maßstab auch auf andere Inseln des Archipels exportiert wurde, als es noch keinen Zement auf den Kanaren gab. An der Südküste von Jandia lagen mehrere Kalköfen, deren Ruinen heute noch zu sehen sind. Bei Montaña Cardones nördlich von Jandia haben wir von älteren Personen erfahren, dass Euphorbia canariensis wie viele andere Pflanzen in Öfen verheizt wurde, in den Notzeiten des spanischen Bürgerkriegs sogar zum Brotbacken. Natürlich sind sukkulente Euphorbien nicht das beste Brennmaterial, aber der Mangel an Holz zwang die Einwohner, alles Brennbare zu verwerten. Den Aussagen zufolge wurde E. canariensis mit Steinwürfen „bearbeitet“, das Austreten von Milchsaft an vielen Stellen schwächte die Pflanzen so stark, dass sie schließlich vertrockneten und per Esel- oder Dromedar-Lasten weggebracht werden konnten. Ohne dass bisher Daten vorliegen, kann nicht ausgeschlossen werden, dass die Vernichtung des von Burchard fotografierten Bestandes von E. handiensis am leicht zugänglichen Südhang des Cuchillo del Palo zum Teil auf die Verwendung der Pflanzen in Kalkbrennöfen zurückgeht. Man müsste nachforschen, bis wann z. B. der ca. $3 \mathrm{~km}$ von diesem ehemaligen Standort der Art entfernte Kalkofen bei Playa de la Señora in Betrieb gewesen ist und ob sich eventuell Personen finden lassen, die dies noch erlebt haben. Allerdings ist es von der Wolfsmilch-Population des Gran Valle nur halb so weit zu diesem Kalkofen, aus 
bestimmten Gründen (vielleicht, weil die Bestände größer und dichter und somit ergiebiger waren) könnte aber das Heranbringen des Brennmaterials vom Cuchillo del Palo vorgezogen worden sein.

\section{Vorstellungen zum Schutz der Jandia-Wolfsmilch}

Weite Areale mit natürlichen Beständen von $E$. handiensis wurden zerstört, andere sehen bei den gegenwärtig ungenügenden Schutzmaßnahmen diesem Schicksal entgegen. Besonders kritisch ist die Lage in den unteren Teilen sowohl des Gran Valle als auch des Valle de Jorós. Eine kürzlich vorgenommene Zustands-Erhebung zeigt eine ständig abnehmende Zahl von Pflanzen (Lawant \& Suntjens 2006), was wir durch eigene Beobachtungen bestätigen können. Durch diese sich abzeichnenden mehr oder weniger ausgedehnten lokalen Auslöschungen werden die Bestände in den nächsten Jahren weiter zerteilt werden und die genetische Vielfalt der Art wird abnehmen.

Drastische und vielleicht etwas voreilige Maßnahmen, wie die Einzäunung des gesamten Areals von E. handiensis im noch weitgehend intakten Habitat Valle de los Mosquitos, sind aus verschiedenen Gründen, wie schon Lawant \& Suntjens (2006) bei ihrem Besuch des Präsidenten der Inselverwaltung feststellten, nicht durchführbar. Das Land ist zwar in den Naturpark Jandia eingegliedert, aber vollständig im Privatbesitz einer großen Baufirma aus Gran Canaria, die mit solchen Maßnahmen einverstanden sein müsste. Außerdem würden sie auf Widerstand der Bevölkerung stoßen, erst recht bei der heutigen schlechten Wirtschaftslage. Kleinere Einzäunungen, wie das im Jahr 2000 von der Gemeinde Pájara eingerichtete, ca. 3 ha große Schutzgebiet am Südhang des Pico de la Zarza im oberen Teil des Jandia-Gebirges, in dem versuchsweise ab 2006 erfolgreiche Aufforstungen mit Bäumen der Lorbeerwaldzone gemacht wurden und das darüber hinaus dem Schutz des seidenhaarigen Goldsterns dient (Asteriscus sericeus, ein Endemit Fuerteventuras, der auch als Zierpflanze beliebt ist), würden dagegen eher durchführbar sein.
Auch auf anderen Inseln der Kanaren haben sich kleine, gut kontrollierbare Schutzgebiete bewährt.

Die etwa 50 registrierten Viehzüchter Jandias, die zusammen bis zu 15000 Ziegen und Schafe halten, von denen sich aber nicht alle ständig im Gelände befinden, haben z. T. angestammte Weiderechte. Jandia war wahrscheinlich zur Zeit der Ureinwohner eine Art Allmende, die von allen Einwohnern der Insel genutzt werden konnte und in die vor allem in regenarmen Jahren Herden aus anderen Gebieten getrieben wurden, weil die Tiere in den feuchteren Höhenzügen des Gebirges immer etwas Nahrung fanden. Diese Einstellung hat sich bis in unsere Zeit gehalten; heute dient die freie Ziegenhaltung in Jandia, die von verschiedenen Verwaltungsebenen aus subventioniert wird, zum großen Teil nicht der Erzeugung von Milch und Käse, bei der die Tiere in Gehegen gehalten werden, sondern der Fleischproduktion (vor allem Zicklein). Sie ist meist eine Nebentätigkeit, die eine starke, die Gemeinschaft einigende Wirkung der Selbstbestätigung gegenüber modernen, die Traditionen schwächenden Einflüssen ausübt. Ethnologen haben den mehrere Male im Jahr stattfindenden Abtrieb der Ziegen aus dem Gebirge zwecks Markierung und tiermedizinischer Inspektion, der eine Art Volksfest darstellt, sogar als „die bedeutendste ethnographische Manifestation des Kanarischen Archipels" bezeichnet. Biologen und Bodenkundler auch innerhalb der Administration weisen dagegen schon lange auf die negativen Folgen der freien Ziegenhaltung für die Pflanzenwelt und den Boden hin. Manche Tiere, wie die Kanaren-endemische Unterart des Schmutzgeiers (Neophron percnopterus subsp. majorensis), die nur noch auf Fuerteventura eine nennenswerte Population besitzt, profitieren aber von dieser extensiven Form der Ziegenhaltung, sodass es hier zu Widersprüchen bei der Naturschutzplanung kommt (GANGOso et al. 2006). Die Viehzüchter haben eine starke Lobby; ihre Tiere halten sich regelmäßig selbst in den als „zonas de exclusión“ bezeichneten Teilen des Naturparks Jandia auf, also in Ausschluss-Gebieten, die theoretisch nicht bewei- 


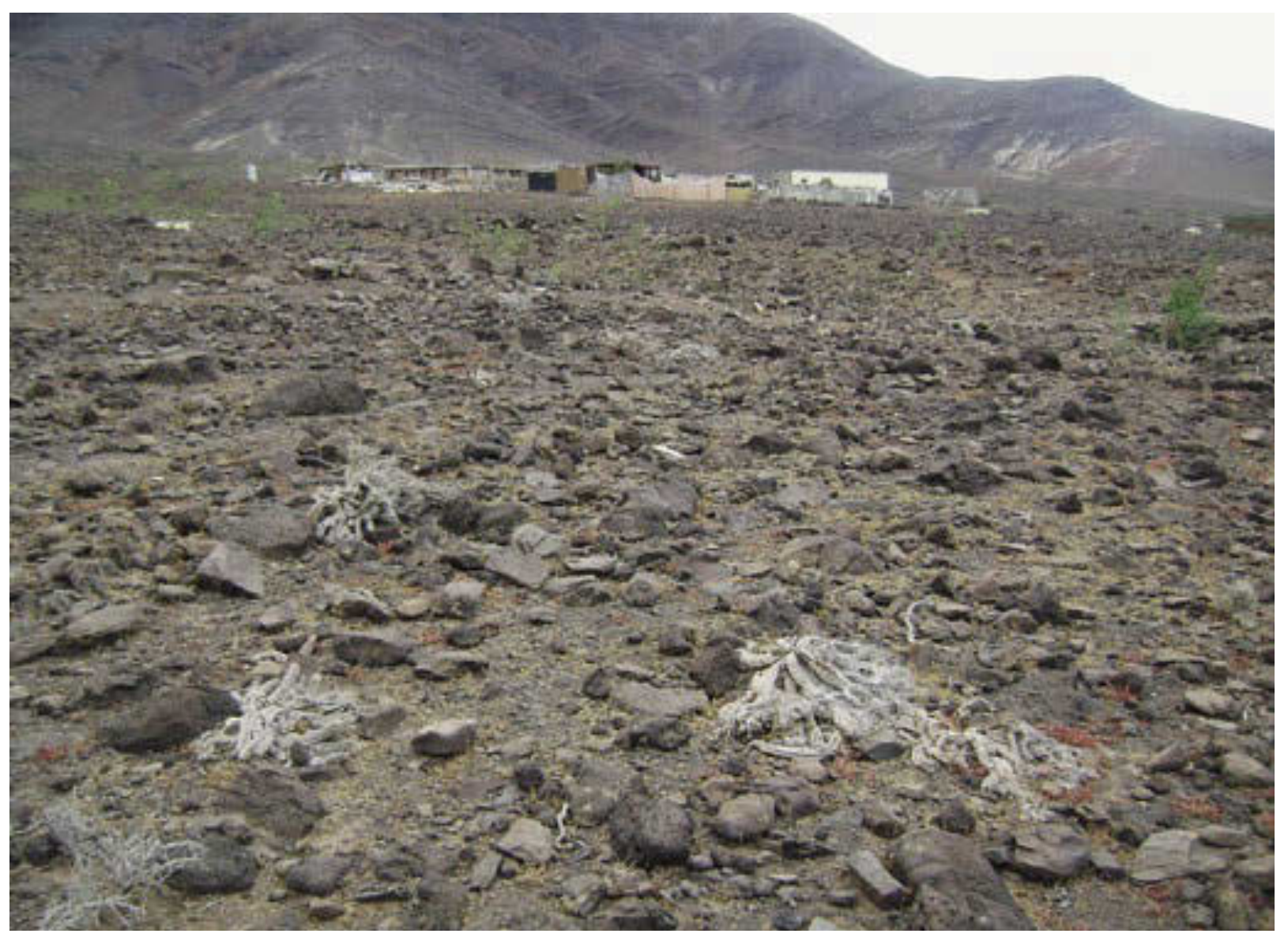

det und nur zu wissenschaftlichen Zwecken betreten werden dürfen. Selbst die Ausweisung Fuerteventuras als UNESCO-Biosphären-Reservat im Jahre 2009 hat daran nichts geändert. Hoffnung ergibt sich im Moment aus der geplanten Einrichtung des Nationalparks Fuerteventura, der große Teile der Westküste der Insel einschließlich des Naturparks Jandia umfassen würde. Sollte er verwirklicht werden, so müsste die Ziegenhaltung in diesen Gebieten zumindest neu reguliert werden, wobei die Interessen des Naturschutzes im Vordergrund zu stehen hätten.

Wir meinen, dass möglichst bald eine umfassende, rigorose Untersuchung der gesamten Problematik stattfinden muss, vielleicht im Rahmen einer oder mehrerer Dissertationen. Die Universität La Laguna auf Teneriffa hat sowohl ein Botanisches als auch ein Pflanzen-

Abb. 9: Abgestorbene und ausgeblichene Euphorbien am Viehzuchtbetrieb La Solana.
schutz-Institut; natürlich sind auch internationale Doktoranden willkommen. Die Arbeiten sollten von der Kanarischen Regionalregierung und von der Inselverwaltung Fuerteventuras finanziell unterstützt werden; immerhin ist E. handiensis offiziell "Simbolo vegetal de Fuerteventura“. Besondere Schwerpunkte müssten sowohl die Untersuchung der Rolle von Pilzen und anderen Pathogenen als auch mögliche Veränderungen in der Bodenchemie und -physik in Gebieten hoher Ziegendichte sein. Kleinere eingezäunte Versuchsparzellen, die aber über eine Reihe von Jahren bestehen müssten, wären leichter zu vertreten als ein großes eingezäuntes Gebiet. Sobald gesicherte Schlussfolgerungen vorlägen, wären vielleicht auch die nötigen Maßnahmen zur Erhaltung der Wolfsmilch-Populationen leichter durchzusetzen, eingeschlossen auch, falls unumgänglich, die Einzäunung größerer Gebiete oder die drastische Reduzierung der Viehbestände.

Wir sind weiterhin dafür, die Standorte der Jandia-Wolfsmilch im Gran Valle und Valle de 
Jorós keineswegs aufzugeben, sondern Untersuchungen und Schutzmaßnahmen gerade hier zu konzentrieren, weil das Überleben dieser Teilpopulationen wichtig ist für die genetische Variabilität von $E$. handiensis und für das Fortbestehen einer ganzen Lebensgemeinschaft aus Pflanzen und Tieren. Ihr Verlust wäre ein trauriger weiterer Schritt in Richtung Desertifikation.

Bemerkenswert ist, dass fast jeder Botanische Garten Mitteleuropas in seiner Sukkulentensammlung eines oder mehrere Exemplare von E. handiensis aufweist, manchmal auch die habituell ähnlichen Verwandten aus Afrika. Auch hierdurch wird ein Beitrag zur Erhaltung bedrohter Euphorbia-Arten geleistet. Aber auch das ist nicht garantiert: durch überregional verordnete Einsparungsmaßnahmen, Streichung oder Rationalisierung von Personalstellen und andere Unwägbarkeiten ist die Unterhaltung von traditionellen Pflanzensammlungen mancherorts nicht mehr gewährleistet. Die Verschiebung wissenschaftlicher Interessen führt oft zu einem Wechsel von Forschungsrichtungen, die traditionelle Pflanzensammlungen für überflüssig erachten, was im Extremfall das Eliminieren des „überflüssigen Pflanzenmaterials“ zugunsten von aktuell angesagten Versuchsflächen und -methoden zur Folge hat. Auch in den Botanischen Gärten könnte E. handiensis somit gefährdet sein.

Um die Vielzahl von Schutzmaßnahmen, die unbedingt getroffen werden müssten, zu vervollständigen, sei abschließend noch ein gefährdetes Tier genannt. Es handelt sich um die endemische Unterart der Kragentrappe Clamydotis undulata fuerteventurae. Der mittelgroße Laufvogel kommt nur auf den Ostinseln vor, sein Lebensraum sind offene, trockene und felsige Landschaften und Kulturland. Auf Fuerteventura soll es nur noch etwa 300 Exemplare geben. Dieser Vogel ist ebenso wie Euphorbia handiensis und die Capra sp. (Ziege) Symbol,
Wahrzeichen und Wappentier von Fuerteventura. Man möge uns eine Schlussbemerkung erlauben, wenn wir den Leser bitten, darüber nachzudenken, wer aus diesem „Dreigespann“ unter den gegenwärtigen Umständen die besten Überlebenschancen haben wird.

\section{Literatur}

Beltrán Tejera, T. E., Wildpret De La Torre, W., Catalina León Arencibia, M., Garcia Gallo, A. \& Reyes Hernandés, J. I999: Libro rojo de la flora canaria contenida en la directiva-habitats europea. - La Laguna, Tenerife.

Bramwell, D. \& Bramwell, Z. 1974: Wild flowers of the Canary islands. Exmo. Cabildo Insular de Tenerife. London.

Burchard, O. 1912: Über eine neue cactoide Euphorbia der Kanarischen Inseln. - Bot. Jahrb. Syst. 48, Beibl. 107: $14-16$.

Burchard, O. 1929: Beiträge zur Ökologie und Biologie der Kanarenpflanzen. - Bibl. Bot. 98. - Stuttgart.

Gómez Campo, C. 1996: Libro rojo de especies vegetales amenazadas de las islas Canarias. Consejeria de politica territorial. - Santa Cruz de Tenerife.

Dominguez, D. M., Reina, M., Santos-Guerra, A., Santana, O., Agulló, T., López-Balboa, C. \& GonzÁlez-Coloma, A. 2008: Pyrrolizidine alkaloids from Canarian endemic plants and their biological effect. - Biochem. Syst. Ecol. 36: 153-166.

Gangoso, L., Donazár, J. A., Scholz, S., Palacios, C. J. \& Hiraldo, F. 2006: Contradiction in conservation of islands ecosystems: plants, introduced herbivores and avian scavengers in the Canary islands. - Biodiv. Conserv. 15: 2231-2248.

Grasmück, H. \& Scholz,S. 2013: 100 Jahre Euphorbia handiensis, Teil I: Entdeckung und Verbreitung auf der Kanareninsel Fuerteventura. - Palmengarten 77: 10-16.

Hansen, H. 1997: Todesursache unbekannt! Zur

Giftigkeit von Senecio-Arten. - Kakteen und andere Sukkulenten 48: 157-159.

Kunkel, G. 1977: Endemismos canarios. Inventario de las plantas vasculares endémicas en la provincia de Las Palmas. - ICONA Monografias 15. - Madrid.

Lawant, P. J. \& Suntjens, R. 2006: Euphorbia handiensis: Will it exist in the future? Euphorbia World 1: 18-29. MagnANI, R. 1992: Euphorbia handiensis BURCHARD in Fuerteventura. - Euphorbia Journal 8: 21-31. Marrero, M. V. \& Carqué, E. 2003: Euphorbia handiensis. - In: Bañares, A. G., Blanca, J., Güemes, J., Moreno, O. \& Ortiz, S. 2003: Atlas y libro rojo de la flora vascular amenazada de España. Direccion general de conservación de la naturaleza. - Madrid. 